\title{
Somatotopic organization of the human spinothalamic tract: in vivo computed tomography-guided mapping in awake patients undergoing cordotomy
}

\author{
C. Michael Honey, MD, ${ }^{1}$ Zurab Ivanishvili, MD, ${ }^{2}$ Christopher R. Honey, MD, DPhil, ${ }^{2}$ and \\ Manraj K. S. Heran, MD ${ }^{3}$ \\ 1Section of Neurosurgery, University of Manitoba, Winnipeg, Manitoba; ${ }^{2}$ Division of Neurosurgery, University of \\ British Columbia, Vancouver, British Columbia; and ${ }^{3}$ Department of Radiology, University of British Columbia, Vancouver, \\ British Columbia, Canada
}

\begin{abstract}
OBJECTIVE The location of the human spinothalamic tract (STT) in the anterolateral spinal cord has been known for more than a century. The exact nature of the neuronal fiber lamination within the STT, however, remains controversial. After correlating in vivo macrostimulation-induced pain/temperature sensation during percutaneous cervical cordotomy with simultaneous CT imaging of the electrode tip location, the authors present a modern description of the somatotopy of the human cervical STT.
\end{abstract}

METHODS Twenty patients underwent CT-guided percutaneous cervical cordotomy to alleviate contralateral medication-refractory cancer pain. Patient responses to electrical stimulation $(0.01-0.1 \mathrm{~V}, 50 \mathrm{~Hz}, 1 \mathrm{msec})$ were recorded and the electrode location for each response was documented with a contemporaneous CT scan. In a post hoc analysis of the data, the location for each patient's response(s) was measured and drawn on a diagram of their cord. Positive responses were represented only when the lowest possible voltage $(\leq 0.02 \mathrm{~V})$ elicited a response. Negative responses were recorded if there was no clinical response at $0.1 \mathrm{~V}$.

RESULTS Clinically, patients did well with an average reduction in opiates of $75 \%$ at 1 week, and $67 \%$ were able to leave the palliative care unit. The size of the cervical cord varied between patients, with an average lateral extent (width) of $11 \mathrm{~mm}$ and a height of $9 \mathrm{~mm}$. Responses from the lower limb were represented superficially (lateral) and posteriorly within the anterolateral cord. The area with responses from the upper limb was larger and surrounded those with responses from the lower limb primarily anteriorly and medially, but also posteriorly.

CONCLUSIONS In this study, the somatotopic organization of the human STT was elucidated for the first time using in vivo macrostimulation and contemporaneous CT imaging during cordotomy. In this cohort of patients, the STT from the lower-limb region was located superficially and posteriorly in the anterolateral quadrant of the cervical cord, with the STT from the upper-limb region surrounding it primarily anteriorly and medially (deep) but also posteriorly. The authors discuss how the previous methods of cordotomy may have biased the earlier versions of STT lamination. They suggest that an ideal spinal cord entry site for cordotomy of either the upper- or lower-limb pain fibers is halfway between the equator and anterior pole of the cord.

https://thejns.org/doi/abs/10.3171/2018.11.SPINE18172

KEYWORDS cordotomy; cancer pain; spinothalamic tract; in vivo mapping

$\mathrm{T}$ HE location of the human spinothalamic tract (STT) in the anterolateral spinal cord has been known for more than a century. ${ }^{3}$ The exact nature of the neuronal fiber lamination within the STT, however, remains controversial. Neurosurgeons continue to describe two very different models of somatotopic organization of the STT: medial-to-lateral ${ }^{4-6,16,24,25}$ and anterior-to-posterior lamina- tion. ${ }^{9,11,13,17,23}$ In the medial-to-lateral model, fibers carrying nociceptive information from the upper limbs are reported to be located in a medial (or deep) location within the tract, whereas fibers from the lower extremities are thought to be located more laterally (or superficially). Neurosurgeons who adhere to this model advocate lesioning deeper within the STT to achieve analgesia in the upper body, and more

ABBREVIATIONS AP = anterior-posterior; $\mathrm{STT}=$ spinothalamic tract. 
superficial lesioning for the lower body. In the anterior-toposterior model, the fibers from the lower limbs are reported to be located in a posterior location within the tract, whereas fibers from the upper extremities are located more anteriorly. According to this model, more posterior lesions would eliminate pain from the lower part of the body, whereas more anterior lesions would eliminate pain from the upper part of the body. In our experience with patients undergoing cordotomy, these models can account for most but not all of the clinical findings during the cordotomy procedure. After correlating in vivo macrostimulation-induced pain or temperature sensation during percutaneous cervical cordotomy with simultaneous CT imaging of the electrode tip location, we present a modern description of the somatotopy of the human cervical STT.

\section{Methods}

The University of British Columbia Clinical Research Ethics Board approved this study. Patients with medically refractory, unilateral, nociceptive pain due to malignancy received contralateral cervical percutaneous cordotomy. Prior to surgery, all opiate medications were converted to an intravenous fentanyl infusion to facilitate rapid postoperative reduction. Procedures were performed under local anesthetic with the patient supine in the CT scanner with monitored anesthetic care. After a skin preparation of the lateral neck, contrast (iohexol $300 \mathrm{mg} / \mathrm{ml}$; GE Healthcare) was initially injected into the posterior cervical thecal sac at the $\mathrm{C} 1-2$ level in order to visualize the cord. Equal volumes of CSF were removed to keep intrathecal pressure neutral. A 300- $\mu \mathrm{m}$-diameter, 3-mm exposed Rosomoff electrode (Radionics) was then inserted percutaneously into the patient's STT contralateral to the pain, with impedance monitoring $(\mathrm{CSF}<200 \Omega$, cord $>400 \Omega)$. Patient responses to electrical stimulation $(0.01-0.1 \mathrm{~V}, 50 \mathrm{~Hz}, 1$ msec) performed using a Radionics RF1A lesion generator (Cosman) were recorded and the electrode location for each response was documented with a contemporaneous CT scan. Ipsilateral motor responses $(0-1.0 \mathrm{~V}, 2 \mathrm{~Hz}, 1$ $\mathrm{msec}$ ) were also tested to determine proximity to the corticospinal tract, but results were not relevant to this study. Axial CT scans were performed with a Siemens Somaton Sensation 16 scanner. Six contiguous $1.5-\mathrm{mm}$ axial slices (using "bone windows" $120 \mathrm{Kv} / 50 \mathrm{~mA} / 0.5 \mathrm{sec}$ rotation) were taken through the region of interest (between $\mathrm{C} 1$ and $\mathrm{C} 2$ ) and stored in a PACS (Picture Archiving and Communication System). Once the appropriate region of the STT was localized, one or more radiofrequency lesions (gradually increasing as needed from 20 to $40 \mathrm{~mA}$ for $60 \mathrm{sec}-$ onds) were made until pinprick appreciation was lost rostral and caudal to the region of pain. The fentanyl infusion was immediately reduced by $50 \%$ postprocedure, and then further reduced, and the patient was transitioned to oral medications by the palliative care team prior to discharge.

In a post hoc analysis of the data, each individual's cervical spinal cord was measured from the CT image by using PACS software. The location of the electrode tip during each stimulation-induced response was then superimposed on the diagram of their cord. Patients often had several responses recorded at different depths during one electrode
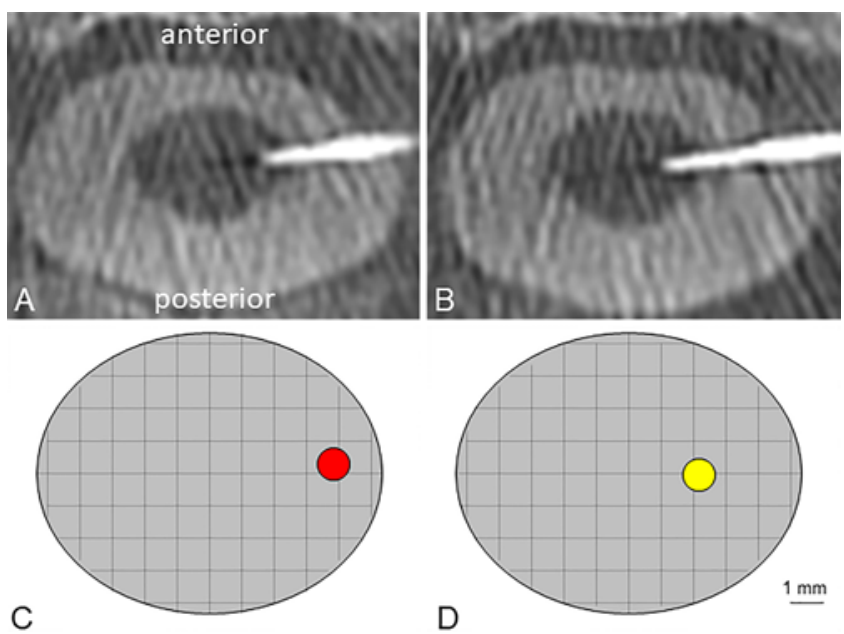

FIG. 1. Electrode location during two stimulation trials in the same patient. Axial CT images of the electrode in the lateral $(\mathbf{A})$ and more medial (B) cervical cord, where macrostimulation ( $\leq 0.02 \mathrm{~V}, 50 \mathrm{~Hz}, 1 \mathrm{msec}$ ) resulted in sensations of "heat" in the patient's contralateral lower limb and upper limb, respectively. The anterior (ventral) cord is shown superiorly. The size of the cord and location of the electrode tip was then measured and shown in panels $\mathbf{C}$ and $\mathbf{D}$, respectively. The effective spread of electrode stimulation was estimated to be approximately $1 \mathrm{~mm}$ from the tip of the electrode because stimulation at position $C$ caused only a lower-limb response (red) and no upper-limb response (yellow). Similarly, stimulation at position $\mathrm{D}$ caused only an upper-limb response.

trajectory into their cord. An example of one trajectory with two different responses at different depths is shown in Fig. 1. Positive responses were represented only when the lowest possible stimulation threshold $(\leq 0.02 \mathrm{~V})$ elicited a response. Responses considered to represent stimulation of the STT were either a reproducible, stimulation-induced pain or a temperature sensation in the contralateral body. Responses in the upper limb were coded as yellow circles on the diagram and responses in the lower limb were coded as red circles. Negative responses were represented when $0.1 \mathrm{~V}$ failed to elicit a clinical response and were coded as gray circles. Each response circle was drawn with a 1-mm diameter on the representative diagram for reasons highlighted below.

All responses were then superimposed onto a representative image of the human cervical spinal cord, the "super cord," with an elliptical shape of $11 \mathrm{~mm}$ lateral extent (width) and $9 \mathrm{~mm}$ height (Fig. 2). Each response location was transferred to fit onto the super cord by maintaining its relative position within the cord (i.e., maintaining the same percentage from the midline to the lateral edge of cord, and from the anterior to posterior edge of cord). The location was reflected horizontally if needed so that all responses were conveniently shown on one side of the cord.

\section{Results}

Twenty patients completed the study. A total of 64 data points were available for analysis with simultaneous clinical responses $(n=41$ positive and $n=23$ negative) and CT-documented electrode tip location. Of the 41 positive responses, 28 occurred following stimulation at the lowest possible threshold of $\leq 0.02 \mathrm{~V}(50 \mathrm{~Hz}, 1 \mathrm{msec})$ and formed 


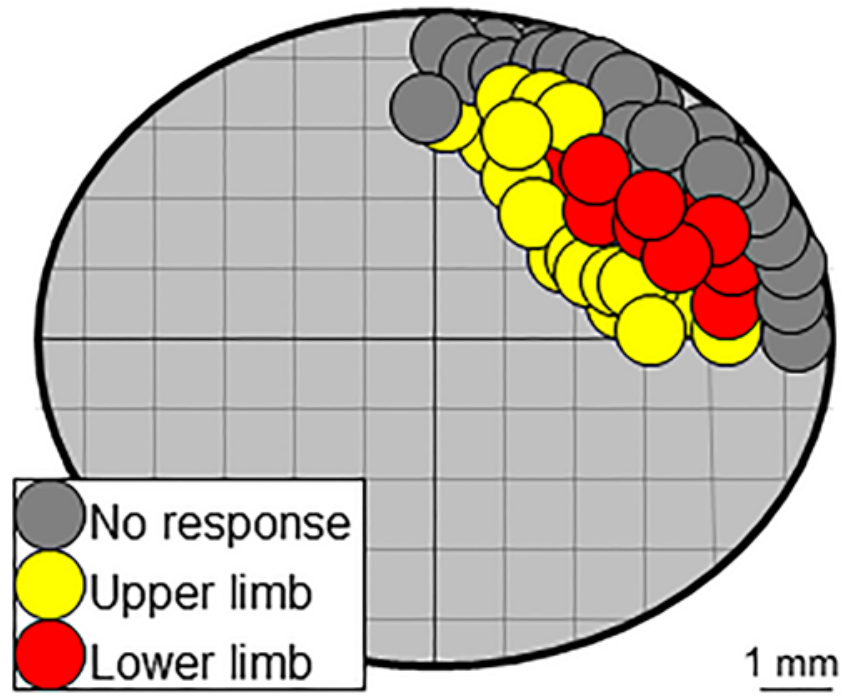

FIG. 2. Location of all responses from the STT superimposed on a super cord. All positive responses from the lower limb (red) and upper limb (yellow) and all negative responses (gray) are located on one side of the super cord. The anterior (ventral) cord is represented superiorly.

the basis for this study. The positive responses from higher thresholds $(0.03-0.1 \mathrm{~V})$ were excluded because they could not be used for definitive location of the components within the STT. The responses were most often reported as "heat" or "pain" and rarely as "cold." Responses anywhere from the hand to the shoulder (C4-T1) were coded as "upper limb," whereas responses anywhere from the hip to the perineum (L1-S5) were coded as "lower limb." Isolated responses in the thorax were rare and were excluded.

Clinically the patients did well. Maximal daily pain on the contralateral side was significantly reduced $(\mathrm{p}<0.001$, paired t-test) from a preoperative average of $9.3 \pm 0.5$ (mean \pm SD measured on a visual analog scale) to $0.5 \pm 1.2$ on the first postoperative day. No benefit in pain on the ipsilateral side was seen. In fact, ipsilateral pain occasionally increased (temporarily) during the aggressive postoperative weaning of opiates. This unmasking of ipsilateral pain was treated with appropriate adjustment of pain medications. From a quality-of-life perspective in our cohort of 20 patients, 12 of the 18 palliative care patients were able to leave the hospital and be cared for at home or in a hospice, and the other 2 patients were treated as outpatients and did not require Palliative Care Unit admission. The average reduction in opiate medications, as measured by morphine equivalents, was $75 \%$ over 1 week (range $40 \%-98 \%$ ). Two patients had a permanent complication: $4+/ 5$ Medical Research Council grade weakness in the ipsilateral arm of a patient who was able to ambulate and go home, and urinary retention requiring an indwelling catheter in a patient with bladder cancer. Temporary complications included urinary retention (1 patient), ipsilateral weakness (2), and bilateral sixth nerve palsy from CSF leakage (1). All reported temporary ipsilateral neck pain, but none developed Ondine's curse (inability to breathe while asleep).

The size and shape of the cervical cord varied remarkably between patients. Most cords were ovoid with a longer maximum lateral extent (width) than anterior-posterior (AP) extent (height), but some were almost circular. The lateral extent ranged from 9.8 to $12.8 \mathrm{~mm}$ and the AP ranged from 7.8 to $10 \mathrm{~mm}$. The average size was $11 \mathrm{~mm}$ lateral and $9 \mathrm{~mm} \mathrm{AP}$, and these dimensions were therefore used for the super cord.

Electrode trajectories near the extreme anterior cord ( $\leq$ $1 \mathrm{~mm}$ from pia mater) caused the cord to rotate away from the puncture site. These trajectories did not produce a positive response because the electrode tip was just beneath the pia and therefore too anterior and superficial to stimulate the STT. Occasionally the cord was deformed by the puncture of the electrode-causing a reduction in cord width and an increase in its height. This change in cord anatomy was never more than $1 \mathrm{~mm}$, and the resultant electrode tip location was based on the postpuncture cord anatomy. The cord was occasionally remarkably mobile within the thecal sac, with 5-mm advances of the electrode just pushing the cord laterally instead of penetrating it-this was suggested by the impedance measurements and confirmed by the CT imaging.

The clinical response following macrostimulation with our equipment at very low voltage could be quite different with advancement of the electrode a further $2 \mathrm{~mm}$ into the cord (i.e., sensation was induced in the leg initially and then in the arm at the greater depth, as seen in Fig. 1). This suggests that the clinical effect of stimulation with our paradigm (0.01-0.02 V, $50 \mathrm{~Hz}, 1 \mathrm{msec})$ extended somewhat less than $2 \mathrm{~mm}$. This is why the response to stimulation was estimated to be approximately $1 \mathrm{~mm}$ for our diagram of the super cord. The expected electrical field surrounding the sharpened tip of a macroelectrode is known to be most dense at the tip, ${ }^{2}$ and hence our diagrams were constructed to show the clinical response in an area of the cord equivalent to a 1-mm-diameter circle around the tip of the electrode.

Figure 2 shows all the data points superimposed onto one representative super cord. The area corresponding to lower-limb responses (red) was smaller than that for the upper limb (yellow). The lower-limb responses were found more superficially and posteriorly to those of the upper limb. Interestingly, the region for upper-limb responses surrounded that for lower-limb responses primarily anteriorly and medially (deep) but also posteriorly.

\section{Discussion}

\section{Historical Perspective}

Brown-Séquard argued that hemisection of the spinal cord caused loss of nociception contralateral to the lesion as part of the syndrome that now bears his name. ${ }^{1}$ This was the first suggestion that different sensory modalities were carried up the cord in anatomically distinct pathways. Toward the end of the 19th century, Gowers postulated that "Thus there is strong reason to regard the antero-lateral ascending tract as containing the path for sensibility to pain." " In a case that would later inspire the cordotomy procedure, Spiller reported in 1905 on a patient with loss of pain sensation below a tuberculosis-induced anterior cord lesion. ${ }^{18} \mathrm{He}$ later convinced Miller to perform the first open thoracic cordotomy in a patient with intractable pain from a spinal tumor. ${ }^{19}$ The operation was successful and quickly gained popularity. Edinger had originally described what 
would become known as the STT (Vorderseitenstränge) in human fetal specimens a few decades ${ }^{3}$ earlier, and this monosynaptic pathway from the spinal cord to the thalamus became recognized as the pathway for nociception interrupted by cordotomy.

Originally, cordotomy was performed as an open thoracic procedure cutting the anterolateral cord (called "chordotomy" at the time because a line was drawn between two points on a circle) after cutting the dentate ligament to rotate the anterior cord into view from a posterior laminectomy approach. Surgeons began to recognize that different depths of the cut resulted in different anatomical extent of analgesia (for review, see Ross ${ }^{16}$ ) and later correlated these effects with postmortem histological findings in the cord. In 1932, Foerster and Gagel first diagrammed the STT with a superficial to deep lamination, with the caudal dermatomes represented more superficially within the cord. In their words, “... je tiefer man dann mit dem Chordotom eindringt, um so höher steigt die Analgesie empor...." (the deeper you penetrate with the chordotomy knife, the higher the analgesia rises). ${ }^{4}$ Later, Hyndman and Van Epps proposed the AP model with the caudal dermatomes represented more posteriorly in the cord. ${ }^{9}$ The first open cervical cordotomy was reported by Stookey. ${ }^{21}$ The technique became more accepted than either multilevel posterior rhizotomy or frontal leukotomy for pain, and multiple publications highlighting the lamination of the STT soon followed. These two different models of STT lamination, essentially either medial-lateral or AP, continued to be cited by neurosurgeons over the following century. Figure 3 shows representative maps of both models from the 1930s to the present.

The percutaneous cervical approach for cordotomy was first reported in 1963 by Mullan et al. ${ }^{14}$ and the current method of radiofrequency lesioning was introduced by Rosomoff et al. ${ }^{15}$ shortly afterward. This immediately reduced the mortality associated with the open procedure and allowed easier access to higher dermatomes. Up until this time, cordotomy was typically performed for lowerlimb pain via a thoracic approach. Percutaneous cervical cordotomy soon eclipsed the open procedure although both are still performed today.$^{20}$ Kanpolat et al. introduced CT-guided percutaneous cordotomy in 1989 (most surgeons had been using fluoroscopy previously). ${ }^{10}$ This team in Ankara later reported being able to selectively eliminate pain in the arm or leg during a cervical cordotomy by aiming the electrode entry just anterior to the dentate (leg region) or further anteriorly (arm region), in keeping with the AP model..$^{11}$

The method of producing cordotomy may have biased the surgeons' understanding of STT lamination. The surgeon was typically able to adjust the depth of a cut for an open procedure and the AP penetration for a percutaneous procedure. Postoperative clinical correlations may therefore have been forced to be compared with the depth of the cut (i.e., medial-lateral lamination) for open cordotomy and with the AP entry for percutaneous cordotomy. There appears to be a trend toward reporting medial-lateral STT lamination during the open cordotomy era and AP lamination during the percutaneous era, although both models continued to be reported.

\section{STT Lamination}

Our results may shed light on the actual lamination pattern of the STT because, for the first time, we report simultaneous clinical response from macrostimulation in the awake patient and anatomical location from CT imaging. Our model of the somatotopic lamination within the STT is documented in Fig. 2 and diagrammed in Fig. 4. Fibers from the lower limb are more superficial and relatively more posterior than those from the upper limb. No electrode trajectory through the cord ever caused a response from the arm followed by a response from the leg at a deeper point. Responses were either "leg" then "arm" at deeper points during trajectories through the posterior half of the anterior cord or just "arm" during more anterior trajectories. No responses were reported from macrostimulation in the most superficial $1 \mathrm{~mm}$ of the lateral cord. This corresponds with the known location of the ventral spinocerebellar tract. No responses were elicited with macrostimulation medial to the expected location of the ventral horn or in the most anterior $1 \mathrm{~mm}$ of the cord. This argues against the existence of a separate "ventral spinothalamic tract" in humans, ${ }^{23}$ despite being described in the macaque monkey as a separate tract from the lateral STT. ${ }^{12}$

The STT is gradually formed from caudal to rostral as pain and temperature fibers enter the spinal cord and then cross the anterior commissure to ascend in the anterolateral cord. ${ }^{3,26}$ Additional fibers from more rostral dermatomes would therefore cross and abut against fibers already present from lower dermatomes. This may explain why the "arm" region is more medial than the "leg." In our study, the clinical responses from the upper limbs covered a larger area within the STT than those from the lower limbs. This is in keeping with studies of nonhuman primates in which almost twice as many fibers within the cervical STT come from dermatomes rostral to $\mathrm{C} 8$ as from those caudal to L1. ${ }^{8}$ We did not find a reproducible homunculus of these upper-limb fibers (i.e., responses localized to the hand could be found anywhere within the upper-limb regions highlighted in yellow on Fig. 2). None of our patients had an uncrossed STT. This is a very rare but reported occurrence within the cordotomy literature. ${ }^{27} \mathrm{We}$ would predict that those patients might have a similar lamination of their STT.

\section{Cordotomy Technique}

Several important practical guidelines for percutaneous cordotomy can be generated from our model of the human cervical STT lamination. First, a trajectory that produces macrostimulation-induced responses from the upper limb is unlikely to produce lower-limb responses at a greater depth. Second, a trajectory that produces responses from the upper limb may produce lower-limb responses at a more superficial depth. Third, an "ideal entry point" into the cervical spinal cord to contact upper-limb pain fibers would be halfway between the equator of the cord and its anterior pole. Fourth, the same "ideal entry point" can be used for lower-limb pain relief by gradually increasing the lesion size, because the electrode will be closer to the pain fibers from the lower limb than the corticospinal tract. This will produce hemianalgesia and hemithermalgesia caudal to the $\mathrm{C} 4$ dermatome, which is well tolerated by patients. 

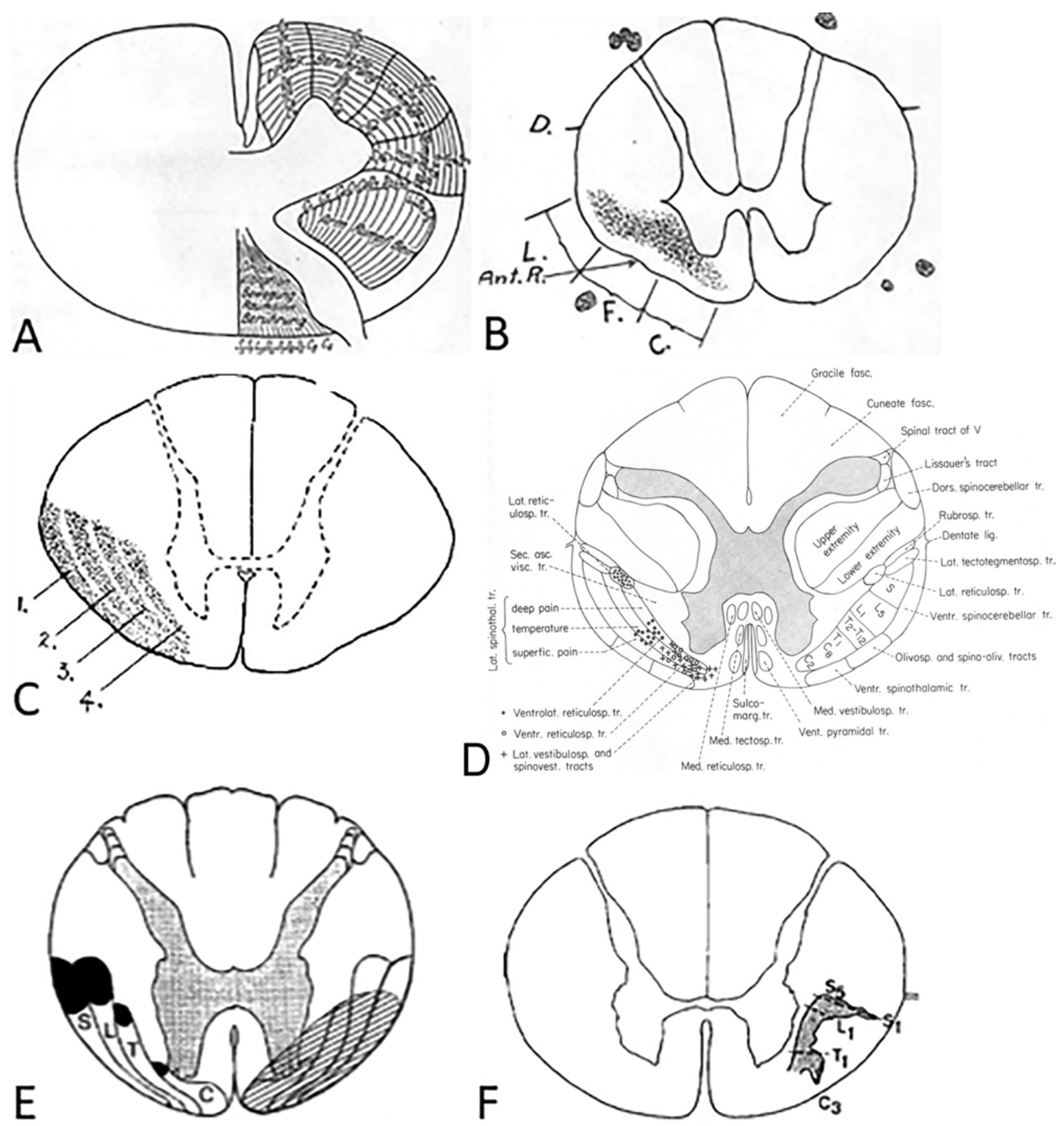

FIG. 3. Representative diagrams of the two models of STT lamination. A: Foerster and Gagel presented the first diagram of STT lamination in 1932 with a deep-to-superficial or medial-lateral orientation for fibers from the upper to lower dermatomes. The anterior (ventral) cervical cord is shown superiorly, whereas in all other diagrams it is shown inferiorly in the anatomical position. Republished with permission of Springer Science and Business Media BV, from "Die Vorderseitenstrangdurchschneidung beim Menschen," Foerster and Gagel, Zeitschrift für gesamte Neurologie und Psychiatrie, vol 138, 1932. Permission conveyed through Copyright Clearance Center, Inc. B: Hyndman and Van Epps introduced the AP model of STT lamination in 1939 with the fibers from the "Lower extremity" (L.) posterior to those from the "Chest" (C.) at the thoracic level. Reproduced with permission from Archives of Surgery, 1939, 38(6):1036-1053. Copyright ( ) (1939) American Medical Association. All rights reserved. C: The medial-lateral model was reported by Glees in 1953 with fibers from the cervical level (labeled "4") medial to those from the sacral level (labeled "1"). Republished with permission of Springer Science and Business Media BV, from "The central pain tract; tractus spinothalamicus," Glees, Acta Neurovegetativa (Wien), vol 7, 1953. Permission conveyed through Copyright Clearance Center, Inc. The medial-lateral model was also reported by Friehs et al. in J Neurosurg 83:8-12, 1995, copyright AANS (E). The AP model is reported by Taren et al. in J Neurosurg 30:569-584, 1969, copyright AANS (D), and by Lahuerta et al. in $J$ Neurosurg 80:975-985, 1994, copyright AANS (F). Panels D-F were published previously in the Journal of Neurosurgery and are reprinted with permission from the AANS. 


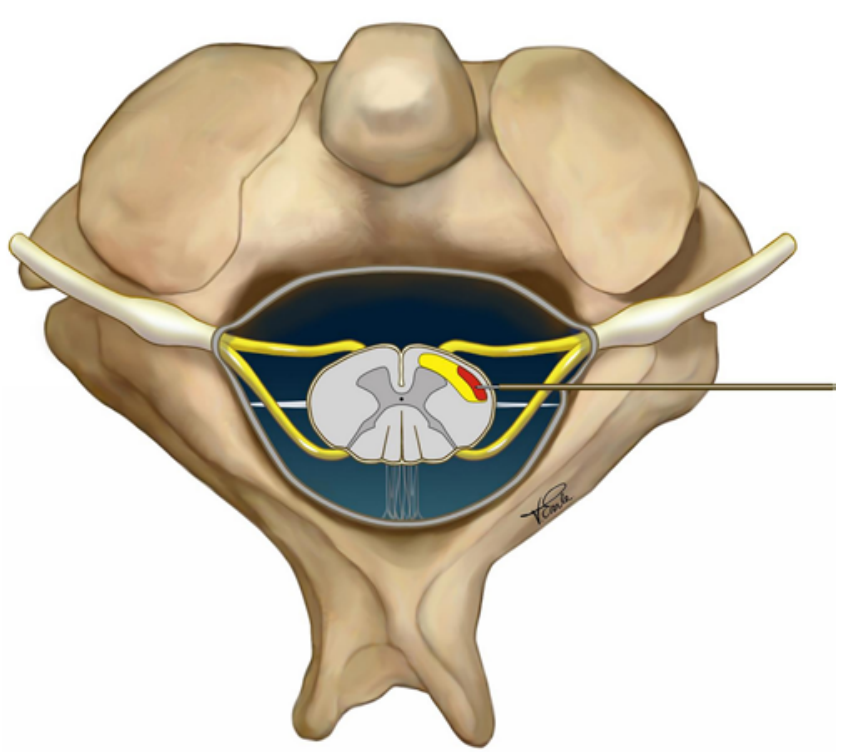

FIG. 4. Model of STT lamination in the human cervical spinal cord. Our results support the theory that the human STT is laminated in the human cervical spinal cord with fibers from the upper limb (yellow) surrounding those from the lower limb (red) primarily anteriorly and medially (deep), but also posteriorly. The spinal cord is drawn in the anatomical (not radiological) position with the posterior (dorsal) surface located inferiorly. Copyright Vicky Earle. Published with permission.

Several important practical guidelines for open cordotomy can also be generated from our model of the human cervical STT lamination. First, fibers from the lower limb are both superficial (as predicted by the medial-lateral model) and posterior (as predicted by the AP model). In order to eliminate pain from more rostral dermatomes, the cut must be both deeper and more anterior. Failure to do both could lead to an inadequate response or so-called islands of pain. Second, the smaller, compact, and more superficial region responsible for pain from the lower limbs may explain why analgesia is more reliably produced in the lower limbs. Third, the unexpected finding of a small portion of pain fibers from the upper limb located more posteriorly (dorsally) to those from the lower limb may explain some cases of residual upper-limb pain, and should guide surgeons to initiate their cut just anterior to the dentate ligament.

\section{Limitations of the Study}

First, we assumed that fibers from the upper and lower limb have a similar clinical response to macrostimulation. If fibers from the upper limb were more sensitive, then a map based on responses to macrostimulation may be artificially enlarged for those fibers. The results highlighted in Fig. 1 argue against this because the clinical responses at each location were confined to either the upper or lower limb and never both when using the lowest possible stimulation setting. Second, we did not distinguish between pain and temperature responses when localizing the STT, although previous authors have suggested that they travel separately. 5,22 Third, patients may have been biased in favor of reporting clinical responses to macrostimulation in the area of their pain because they knew we were search- ing for those fibers. The majority of our patients, however, had pain below the T4 dermatome, whereas the majority of responses to macrostimulation were above that level. Fourth, the unexpected finding of upper-limb fibers as far posterior as the equator of the cord (further posterior than any lower-limb fibers) was only detected in 2 patients and may therefore just be a rare anomaly. Both of those patients had pain in the lower limb, and the electrode was deliberately guided to the most posterior aspect of the anterolateral cord, only to have to be redirected more anteriorly. Fifth, there are pain pathways other than the STT within the anterolateral spinal cord that may have been stimulated by our electrode. It is undoubtedly important to destroy the spinomesencephalic and spinoreticular tracts $^{26}$ during cordotomy, but these tracts do not have the small receptive fields of those STT fibers ending in the lateral thalamus, and therefore typical macrostimulation-induced responses such as "warmth in the hand" or "pain in the hip" probably represented stimulation of the STT. We did not test our patients for acute changes in blood pressure, heart rate, eye movement, alertness, or mood. Finally, our final description of the STT (Fig. 4) represents a probabilistic map or the summary of all responses from a cohort of patients rather than an image for any one patient. As such, the size of the area where the STT could be in our cohort is larger than where it actually is for any given patient. Future studies using diffusion tensor tractography may be able to provide individualized maps of the STT.

\section{Conclusions}

Our work simultaneously combined awake physiological localization of fibers within the human STT with neuroimaging documenting their precise anatomical localization within the spinal cord. The resultant map of the STT demonstrates, for the first time, that fibers from the lower limb are located superficially and posteriorly within the anterolateral cord, with the fibers from the upper limb surrounding them primarily deep and anteriorly but also posteriorly. If these results are confirmed by others, it will solve the century-long controversy of lamination patterns within the STT and add a small but fundamental piece of knowledge to our understanding of human neuroanatomy.

\section{Acknowledgments}

We thank Professor Dr. med. Joachim Krauss for assistance in translation from the original German. The illustration of Fig. 4 was provided by Ms. Vicky Earle.

\section{References}

1. Brown-Séquard CE: Lectures on the physiology and pathology of the nervous system; and on the treatment of organic nervous affections. Lancet 1:1-3, 1869

2. Cosman ER Jr, Cosman ER Sr: Electric and thermal field effects in tissue around radiofrequency electrodes. Pain Med 6:405-424, 2005

3. Edinger L: Vergleichend-entwicklungsgeschichtliche und anatomische Studien im Bereiche des Centralnervensystems: II. Uber die Fortsetzung der hinteren Rückenmarkswurzeln zum Gehirn. Anat Anz 4:121-128, 1889

4. Foerster O, Gagel O: Die Vorderseitenstrangdurchschneidung beim Menschen. Ztschr ges Neurol Psychiat 138:1-92, 1932 
5. Friehs GM, Schröttner O, Pendl G: Evidence for segregated pain and temperature conduction within the spinothalamic tract. J Neurosurg 83:8-12, 1995

6. Glees P: The central pain tract; tractus spino-thalamicus. Acta Neuroveg (Wien) 7:160-174, 1953

7. Gowers WR: A Manual of Diseases of the Nervous System. Vol 1. Diseases of the Nerves and Spinal Cord, ed 2. Philadelphia: P. Blakiston, Son \& Co., 1892, p 198

8. Hodge CJ Jr, Apkarian AV: The spinothalamic tract. Crit Rev Neurobiol 5:363-397, 1990

9. Hyndman OR, Van Epps C: Possibility of differential section of the spinothalamic tract. A clinical and histologic study. Arch Surg 38:1036-1053, 1939

10. Kanpolat Y, Deda H, Akyar S, Bilgiç S: CT-guided percutaneous cordotomy. Acta Neurochir Suppl (Wien) 46:67-68, 1989

11. Kanpolat Y, Ugur HC, Ayten M, Elhan AH: Computed tomography-guided percutaneous cordotomy for intractable pain in malignancy. Neurosurgery 64 (3 Suppl):ons187ons194, 2009

12. Kerr FWL: Neuroanatomical substrates of nociception in the spinal cord. Pain 1:325-356, 1975

13. Lahuerta J, Bowsher D, Lipton S, Buxton PH: Percutaneous cervical cordotomy: a review of 181 operations on 146 patients with a study on the location of "pain fibers" in the C-2 spinal cord segment of 29 cases. J Neurosurg 80:975-985, 1994

14. Mullan S, Harper PV, Hekmatpanah J, Torres H, Dobbin G: Percutaneous interruption of spinal-pain tracts by means of a strontium ${ }^{90}$ needle. J Neurosurg 20:931-939, 1963

15. Rosomoff HL, Brown CJ, Sheptak P: Percutaneous radiofrequency cervical cordotomy: technique. J Neurosurg 23:639_ 644, 1965

16. Ross JP: The anatomy of the spinothalamic tract in relation to cordotomy. Proc R Soc Med 24:975-976, 1931

17. Schneider RJ, Paul RL: Body representation in the anterolateral funiculus as reconstructed by percutaneous cordotomies for benign intractable pain. Adv Pain Res Ther 1:267-270, 1976

18. Spiller WG: The occasional clinical resemblance between caries of the vertebrae and lumbothoracic syringomyelia and the location within the spinal cord of fibres for the sensations of pain and temperature. Univ Penn Med Bull 18:147-154, 1905

19. Spiller WG, Martin E: The treatment of persistent pain of organic origin in the lower part of the body by division of the anterolateral column of the spinal cord. JAMA 58:14891490,1912

20. Steel D, Kirkman MA, Thompson DNP, Aquilina K: Open thoracic anterolateral cordotomy for pain relief in children: report of 2 cases. J Neurosurg Pediatr 20:278-283, 2017

21. Stookey B: Chordotomy of the second cervical segment for relief of pain due to recurrent carcinoma of the breast. Arch Neurol Psychiatry 26:443, 1931

22. Stookey B: Human chordotomy to abolish pain sense without destroying temperature sense. J Nerv Ment Dis 69:552-557, 1929

23. Taren JA, Davis R, Crosby EC: Target physiologic corroboration in stereotaxic cervical cordotomy. J Neurosurg 30:569_ 584,1969

24. Tranmer BI, Tucker WS, Bilbao JM: Sleep apnea following percutaneous cervical cordotomy. Can J Neurol Sci 14:262267, 1987

25. Walker AE: The spinothalamic tract in man. Arch Neurol Psychiatry 43:284-298, 1940

26. Willis WD, Westlund KN: Neuroanatomy of the pain system and of the pathways that modulate pain. J Clin Neurophysiol 14:2-31, 1997

27. Voris HC: Variations in the spinothalamic tract in man. J Neurosurg 14:55-60, 1957

\section{Disclosures}

The authors report no conflict of interest concerning the materials or methods used in this study or the findings specified in this paper.

\section{Author Contributions}

Conception and design: CM Honey, CR Honey, Heran. Acquisition of data: CM Honey, Ivanishvili, Heran. Analysis and interpretation of data: CM Honey, Ivanishvili, Heran. Drafting the article: CM Honey. Critically revising the article: all authors. Reviewed submitted version of manuscript: all authors. Approved the final version of the manuscript on behalf of all authors: CM Honey. Statistical analysis: CM Honey.

\section{Supplemental Information}

Previous Presentations

Portions of this work were presented at the 2018 Canadian Neurological Sciences Federation Congress in Halifax, Nova Scotia, Canada (June 27, 2018).

\section{Correspondence}

C. Michael Honey: University of Manitoba, Winnipeg, MB, Canada.c.michael.honey@gmail.com. 